\title{
Safety Evaluation of Excessive Intake of Lactococcus lactis Subsp. lactis JCM 5805: A Randomized, Double-Blind, Placebo-Controlled, Parallel-Group Trial
}

\section{Yukiko Kato ${ }^{*}$, Masaya Kanayama1, Shu Yanai², Hajime Nozawa², Osamu Kanauchi', Satoru Suzuki ${ }^{3}$}

\author{
${ }^{1}$ Research Laboratories for Health Science \& Food Technologies, Kirin Co., Ltd., Tokyo, Japan \\ ${ }^{2}$ Central Laboratories for Key Technologies, Kirin Co., Ltd., Tokyo, Japan \\ ${ }^{3}$ Shinagawa Season Terrace Health Care Clinic, Tokyo, Japan \\ Email: *Yukiko_Kato@kirin.co.jp
}

How to cite this paper: Kato, Y., Kanayama, M., Yanai, S., Nozawa, H., Kanauchi, O. and Suzuki, S. (2018) Safety Evaluation of Excessive Intake of Lactococcus lactis Subsp. lactis JCM 5805: A Randomized, Double-Blind, Placebo-Controlled, ParallelGroup Trial. Food and Nutrition Sciences, 9, 403-419.

https://doi.org/10.4236/fns.2018.94032

Received: March 13, 2018

Accepted: April 27, 2018

Published: April 30, 2018

Copyright $\odot 2018$ by authors and Scientific Research Publishing Inc. This work is licensed under the Creative Commons Attribution International License (CC BY 4.0).

http://creativecommons.org/licenses/by/4.0/

\begin{abstract}
Background/Aims: Administration of a lactic acid bacterial strain, Lactococcus lactis subsp. lactis JCM 5805 (LC-Plasma), is reported to prevent viral infection via activation of plasmacytoid dendritic cells in mouse and human studies. As it is assumed that LC-Plasma is taken in excess when it is commercially provided as a supplement, we conducted a trial using capsules to give $250 \mathrm{mg}$ LC-Plasma ( 5 times the effective anti-viral dose) every day for four weeks to healthy volunteers to investigate the safety of excessive intake of LC-Plasma. Trial Design: A randomized, double-blind, placebo-controlled, parallel-group trial was conducted. Methods: Forty healthy subjects were randomly assigned to the LC-Plasma group (daily intake of five capsules containing $50 \mathrm{mg}$ heat-killed LC-Plasma cells per capsule) or the placebo group (daily intake of five placebo capsules with no LC-Plasma). Physical, hematological, biochemical and urinary examinations and medical interviews were used to evaluate safety. Results: No abnormal differences were observed after excessive intake of LC-Plasma capsules when compared to the intake of placebo capsules. Conclusions: There are no safety concerns associated with the excessive intake of heat-killed LC-Plasma capsules.
\end{abstract}

\section{Keywords}

Lactic Acid Bacteria, Lactococcus lactis, Excessive Intake, Safety 


\section{Introduction}

Lactic acid bacteria (LAB) have been traditionally used in the production of fermented foods, such as yogurt and cheese, and are regarded as highly safe food materials. It is reported that LAB have many health functions, such as the regulation of intestinal cells, immune cells and microbiota, and they have been used in the production of many functional health foods [1] [2]. We previously found that Lactococcus lactis strain Plasma (LC-Plasma), which is a synonym of Lactococcus lactis subsp. lactis JCM 5805, stimulated and activated plasmacytoid dendritic cells $(\mathrm{pDC})$, regardless of whether they were live or heat-killed [3]. pDC are immune cells, responsible for early reaction to viral infection and play a key role in preventing the proliferation and spread of viruses by production of type I interferons, a key factor in various immune reactions against viruses [4] [5]. We previously reported that LC-Plasma induced the production of interferon- $\alpha($ IFN- $\alpha)$ in an in vitro study [3] and prevented viral infection in mouse and human studies [6]-[11]. In addition, oral administration of LC-Plasma significantly suppressed lung inflammation and death caused by parainfluenza virus infection in mice via enhancement of lung anti-viral immunity [6]. LC-Plasma also alleviated the following symptoms of rotaviral infection: weight loss, aggravation of fecal scores and rotavirus outgrowth in mice via increasing mature pDC in the small intestine [7]. In a clinical trial, the expression levels of anti-viral genes in human peripheral blood mononuclear cells (PBMC) stimulated by inactivated human influenza virus $\mathrm{A} / \mathrm{H} 1 \mathrm{~N} 1(\mathrm{~A} / \mathrm{PR} / 8 / 34)$ were higher in the subjects who took LC-Plasma compared with subjects who did not take LC-Plasma [8]. In other clinical studies, the intake of yogurt or supplement containing $50 \mathrm{mg}\left(1.0 \times 10^{11}\right.$ cells or more $)$ of LC-Plasma activated human $\mathrm{pDC}$ and alleviated the symptoms of flu and common cold [9] [10] [11]. Although it was demonstrated that the intake of $50 \mathrm{mg}\left(1.0 \times 10^{11}\right.$ cells or more $)$ of LC-Plasma did not produce any safety concerns, it is assumed that LC-Plasma is taken in excess when it is provided as a supplement. To investigate the safety of excessive intake of LC-Plasma, we conducted a trial where healthy volunteers ingested capsules containing $250 \mathrm{mg}$ LC-Plasma (5 times the effective anti-viral dose) every day for four weeks.

\section{Subjects and Method}

This trial was approved by the Institutional Review Board in Shinagawa Season Terrace Health Care Clinic (Minato-ku, Tokyo) in accordance with the Declaration of Helsinki and the Ethical Guidelines for Medical and Health Research Involving Human Subjects (approved on April 28, 2017, UMIN test ID: UMIN000027309).

\subsection{Subjects}

This trial was conducted at Shinagawa Season Terrace Health Care Clinic. Healthy male and female adult volunteers (20 to 64 years old) were recruited in 
May 2017 by KSO Corporation. All subjects were provided a sufficient explanation regarding the trial including the trial purpose, contents, methods, and expected adverse events. Written informed consent was obtained from all volunteers before the start of the trial. The exclusion criteria were as follows: excessive alcohol-drinking behavior; unable to abstain from drinking alcohol from two days before each examination; chronic illness and regular use of medication; under treatment or with a history of serious disease (e.g., diabetes, liver disease, kidney disease or heart disease), thyroid gland disease, adrenal gland disease, or other metabolic disorder; any food allergy; under treatment for or with a history of hay fever; under treatment for or with a history of inflammatory bowel disease (e.g., ulcerative colitis or Crohn's disease); history of digestive disease affecting digestion or absorption; frequent diarrhea after ingesting dairy products; under treatment for or with a history of drug addiction or alcoholism; possible pregnancy, pregnancy or lactating; unfavorable blood test result at the baseline period; participation or possible participation in another clinical trial using any other food, medicine or cosmetics during this trial; any other reason for ineligibility as judged by the principal investigator.

Sample size was determined to be twenty subjects for each group: guidelines from the Japan Society of Health and Nutrition Food Association recommend at least twenty subjects when conducting a single safety trial [12].

\subsection{Trial Design}

The trial design was a randomized, double-blind, placebo-controlled, parallel group comparison experiment, conducted from May to July 2017. The baseline period before capsule intake was two weeks, capsule intake period was four weeks, and the follow-up observation period after the completion of intake was two weeks.

Subjects were randomly divided into two groups (the LC-Plasma group or placebo group) containing 20 subjects each by the person responsible for statistical analysis so that no bias was generated in the baseline data (sex, age or BMI). Subjects consumed either five LC-Plasma capsules containing $50 \mathrm{mg}\left(1.0 \times 10^{11}\right.$ or more) of heat-killed LC-Plasma per capsule or five placebo capsules that did not contain LC-Plasma, daily for four weeks. Capsules were consumed at any time of the day during the intake period. Capsules were manufactured under the responsibility of Kirin Co., Ltd. The placebo capsules were made from the same materials and according to the same prescription as the LC-Plasma capsules except that LC-Plasma was replaced with cornstarch. Neither the investigators nor the subjects could distinguish between the capsules. The allocation table was sealed and stored securely by the controller; in addition, the capsule codes were blinded and the data set was locked until all analyses were complete. The examinations included: physical examination (height, weight, body mass index (BMI), systolic blood pressure, diastolic blood pressure, and pulse rate), hematological examination (white blood cell count, red blood cell count, hemoglobin 
$(\mathrm{Hb})$, hematocrit $(\mathrm{Ht})$, mean corpuscular volume (MCV), mean corpuscular hemoglobin $(\mathrm{MCH})$, mean corpuscular hemoglobin concentration (MCHC), platelet count, differential leucocyte count [neutrophils (NEUT), lymphocytes (LYMPH), monocytes (MONO), eosinophils (EOSINO), and basophils (BASO)]), blood biochemical examination (total protein, albumin, total bilirubin, aspartate aminotransferase (AST (GOT)), alanine aminotransferase (ALT (GPT)), lactic dehydrogenase $(\mathrm{LDH})$, alkaline phosphatase (ALP), $\gamma$-glutamyl transpeptidase $(\gamma$-GTP), creatine phosphokinase (CPK), urea nitrogen, creatinine, uric acid, sodium $(\mathrm{Na})$, chlorine $(\mathrm{Cl})$, potassium $(\mathrm{K})$, calcium $(\mathrm{Ca})$, inorganic phosphorus, magnesium, serum iron, total cholesterol, low density lipoprotein (LDL)-cholesterol, high density lipoprotein (HDL)-cholesterol, triglyceride (TG), fasting blood glucose, and HbA1c (NGSP value)), urinalysis (urine protein, urine glucose, urinary bilirubin, urinary ketone body, urinary occult blood reaction, urinary urobilinogen, urine $\mathrm{pH}$, and urine specific gravity), and a medical interview conducted by a physician. Hematological examination, blood biochemical examination and urinalysis were performed by LSI Medience Corporation under consignment.

Capsule intake, physical health, living conditions and the intake of health food products and medicines were listed in the subject's diary from fourteen days before the start of capsule intake (in week 0 ) and until the day before the medical examination during the follow up observation period (in week 6). All meals (including alcohol) ingested during the period from week 0 until week 6 were recorded in a dietary diary. In addition, the contents of meals taken during the period from 7 days before the day of examinations in week 0 , week 2, week 4 and week 6 (28 days in total) were recorded in detail in the dietary diary. The records in the diary were checked on the day of each visit date, and subjects were strictly guided if any violations such as surfeit were found.

\subsection{Statistical Analysis}

The mean and standard deviation were calculated for all parameters measured at the baseline period before intake. Statistical analysis was performed by one-way analysis of variance (ANOVA) and the measured values were tabulated (Table 1(a)). Cross-tabulation tables were prepared for data on gender and qualitative urinalysis, and the results of qualitative urinalysis were scored and analyzed by Wilcoxon's rank sum test (Table 1 and Table 2).

The mean and standard deviation were calculated for all parameters measured in each group. Statistical analysis was performed by paired t-test (two-tailed test) for comparison of the data obtained at the start of ingestion (in week 0), and those obtained at the examination in weeks 2, 4 (intake period) and 6 (at the end of the follow up). In addition, statistical analysis was conducted using unpaired t-tests (two-tailed test) for comparison of the data at each examination between the LC-Plasma group and placebo group (Tables 2-8). Stratified analysis by sex was conducted for the measurement parameters in which the reference values 
Table 1. (a) Characteristics of the study subjects at baseline period; (b) Characteristics of the study subjects at baseline period.

(a)

\begin{tabular}{|c|c|c|c|c|c|}
\hline \multirow{2}{*}{ Parameter } & \multirow{2}{*}{ Unit } & LC-Plasma $(\mathrm{n}=20)$ & Placebo $(\mathrm{n}=20)$ & \multirow{2}{*}{$p$ value } & \multirow{2}{*}{$\begin{array}{c}\text { Whole }(\mathrm{n}=40) \\
\text { mean } \pm \mathrm{SD}\end{array}$} \\
\hline & & mean $\pm S D$ & mean $\pm \mathrm{SD}$ & & \\
\hline Age & years & $44.3 \pm 12.0$ & $43.8 \pm 9.9$ & 0.875 & $44.0 \pm 10.9$ \\
\hline Height & $\mathrm{cm}$ & $164.8 \pm 9.3$ & $165.2 \pm 8.7$ & 0.881 & $165.0 \pm 8.9$ \\
\hline Body weight & $\mathrm{kg}$ & $59.6 \pm 9.2$ & $60.4 \pm 7.9$ & 0.770 & $60.0 \pm 8.5$ \\
\hline BMI & & $21.9 \pm 1.7$ & $22.1 \pm 1.9$ & 0.660 & $22.0 \pm 1.8$ \\
\hline Systolic blood pressure & $\mathrm{mmHg}$ & $119.4 \pm 14.1$ & $121.4 \pm 13.1$ & 0.644 & $120.4 \pm 13.5$ \\
\hline Diastolic blood pressure & $\mathrm{mmHg}$ & $72.6 \pm 11.3$ & $71.7 \pm 10.8$ & 0.786 & $72.1 \pm 10.9$ \\
\hline Pulse rate & bpm & $72.5 \pm 10.5$ & $71.2 \pm 7.8$ & 0.646 & $71.8 \pm 9.1$ \\
\hline White blood cell count & $/ \mathrm{mL}$ & $5640 \pm 1188$ & $6300 \pm 1505$ & 0.132 & $5970 \pm 1380$ \\
\hline Red blood cell count & $\times 10^{4} / \mathrm{mL}$ & $476.6 \pm 44.8$ & $466.1 \pm 36.7$ & 0.423 & $471.3 \pm 40.8$ \\
\hline Hemoglobin & $\mathrm{g} / \mathrm{dL}$ & $14.5 \pm 1.5$ & $14.3 \pm 1.5$ & 0.634 & $14.4 \pm 1.5$ \\
\hline Hematocrit & $\%$ & $44.9 \pm 4.0$ & $43.9 \pm 4.2$ & 0.460 & $44.4 \pm 4.1$ \\
\hline Platelet count & $\times 10^{4} / \mathrm{mL}$ & $27.3 \pm 5.8$ & $25.7 \pm 3.5$ & 0.289 & $26.5 \pm 4.8$ \\
\hline $\mathrm{MCV}$ & $\mathrm{fl}$ & $94.4 \pm 4.5$ & $94.4 \pm 3.2$ & 0.968 & $94.4 \pm 3.9$ \\
\hline $\mathrm{MCH}$ & pg & $30.4 \pm 1.9$ & $30.6 \pm 1.2$ & 0.788 & $30.5 \pm 1.6$ \\
\hline $\mathrm{MCHC}$ & $\%$ & $32.2 \pm 0.9$ & $32.4 \pm 0.7$ & 0.371 & $32.3 \pm 0.8$ \\
\hline NEUT/leukocyte fractionation & $\%$ & $56.3 \pm 9.3$ & $57.6 \pm 7.3$ & 0.615 & $56.9 \pm 8.2$ \\
\hline LYMPH/leukocyte fractionation & $\%$ & $34.7 \pm 7.8$ & $33.6 \pm 6.3$ & 0.608 & $34.2 \pm 7.0$ \\
\hline MONO/leukocyte fractionation & $\%$ & $5.5 \pm 1.4$ & $5.0 \pm 1.0$ & 0.208 & $5.3 \pm 1.2$ \\
\hline EOSINO/leukocyte fractionation & $\%$ & $3.0 \pm 2.3$ & $3.2 \pm 2.3$ & 0.805 & $3.1 \pm 2.3$ \\
\hline BASO/leukocyte fractionation & $\%$ & $0.5 \pm 0.3$ & $0.6 \pm 0.4$ & 0.195 & $0.6 \pm 0.3$ \\
\hline Total protein & $\mathrm{g} / \mathrm{dL}$ & $7.1 \pm 0.4$ & $7.2 \pm 0.4$ & 0.517 & $7.1 \pm 0.4$ \\
\hline Albumin & $\mathrm{g} / \mathrm{dL}$ & $4.4 \pm 0.3$ & $4.4 \pm 0.3$ & 0.604 & $4.4 \pm 0.3$ \\
\hline AST (GOT) & $\mathrm{U} / \mathrm{L}$ & $19.1 \pm 4.0$ & $18.1 \pm 3.3$ & 0.392 & $18.6 \pm 3.6$ \\
\hline ALT (GPT) & $\mathrm{U} / \mathrm{L}$ & $16.1 \pm 7.9$ & $16.1 \pm 5.9$ & 0.982 & $16.1 \pm 6.9$ \\
\hline LD (LDH) & $\mathrm{U} / \mathrm{L}$ & $181.9 \pm 23.0$ & $168.8 \pm 17.2$ & 0.049 & $175.3 \pm 21.1$ \\
\hline Total bilirubin & $\mathrm{mg} / \mathrm{dL}$ & $0.80 \pm 0.26$ & $0.74 \pm 0.21$ & 0.459 & $0.77 \pm 0.23$ \\
\hline ALP & $\mathrm{U} / \mathrm{L}$ & $181.4 \pm 48.4$ & $199.9 \pm 52.8$ & 0.255 & $190.6 \pm 50.8$ \\
\hline$\gamma$-GT $(\gamma$-GTP $)$ & $\mathrm{U} / \mathrm{L}$ & $20.0 \pm 10.0$ & $20.0 \pm 8.9$ & 1.000 & $20.0 \pm 9.4$ \\
\hline CK (CPK) & $\mathrm{U} / \mathrm{L}$ & $116.3 \pm 56.2$ & $96.1 \pm 46.9$ & 0.226 & $106.2 \pm 52.1$ \\
\hline Urea nitrogen & $\mathrm{mg} / \mathrm{dL}$ & $12.7 \pm 3.7$ & $13.0 \pm 3.2$ & 0.779 & $12.9 \pm 3.4$ \\
\hline Creatinine & $\mathrm{mg} / \mathrm{dL}$ & $0.76 \pm 0.18$ & $0.75 \pm 0.11$ & 0.782 & $0.75 \pm 0.15$ \\
\hline Uric acid & $\mathrm{mg} / \mathrm{dL}$ & $5.3 \pm 1.4$ & $5.2 \pm 1.0$ & 0.689 & $5.2 \pm 1.2$ \\
\hline Sodium $(\mathrm{Na})$ & $\mathrm{mEq} / \mathrm{L}$ & $141.3 \pm 1.9$ & $141.4 \pm 1.4$ & 0.848 & $141.3 \pm 1.6$ \\
\hline Chloride $(\mathrm{Cl})$ & $\mathrm{mEq} / \mathrm{L}$ & $104.5 \pm 1.9$ & $104.9 \pm 2.2$ & 0.545 & $104.7 \pm 2.1$ \\
\hline
\end{tabular}




\section{Continued}

\begin{tabular}{cccccc}
\hline Potassium $(\mathrm{K})$ & $\mathrm{mEq} / \mathrm{L}$ & $4.4 \pm 0.3$ & $4.4 \pm 0.3$ & 0.364 & $4.4 \pm 0.3$ \\
Calcium $(\mathrm{Ca})$ & $\mathrm{mg} / \mathrm{dL}$ & $9.7 \pm 0.3$ & $9.6 \pm 0.3$ & 0.151 & $9.7 \pm 0.3$ \\
Inorganic phosphorus & $\mathrm{mg} / \mathrm{dL}$ & $3.3 \pm 0.5$ & $3.5 \pm 0.4$ & 0.230 & $3.4 \pm 0.5$ \\
Magnesium & $\mathrm{mg} / \mathrm{dL}$ & $2.3 \pm 0.1$ & $2.3 \pm 0.1$ & 0.347 & $2.3 \pm 0.1$ \\
Serum iron & $\mathrm{mg} / \mathrm{dL}$ & $109.6 \pm 43.2$ & $101.0 \pm 26.3$ & 0.455 & $105.3 \pm 35.6$ \\
Total cholesterol & $\mathrm{mg} / \mathrm{dL}$ & $200.1 \pm 24.5$ & $200.1 \pm 26.6$ & 0.995 & $200.1 \pm 25.2$ \\
LDL-cholesterol & $\mathrm{mg} / \mathrm{dL}$ & $115.4 \pm 23.0$ & $112.1 \pm 20.7$ & 0.641 & $113.7 \pm 21.7$ \\
HDL-cholesterol & $\mathrm{mg} / \mathrm{dL}$ & $62.3 \pm 13.0$ & $69.3 \pm 16.0$ & 0.137 & $65.8 \pm 14.8$ \\
Triglyceride (TG) & $\mathrm{mg} / \mathrm{dL}$ & $93.7 \pm 49.3$ & $83.7 \pm 45.6$ & 0.509 & $88.7 \pm 47.1$ \\
Fasting blood glucose & $\mathrm{mg} / \mathrm{dL}$ & $84.5 \pm 7.8$ & $82.8 \pm 5.6$ & 0.433 & $83.7 \pm 6.7$ \\
HbAlc & $\%$ & $5.5 \pm 0.3$ & $5.4 \pm 0.3$ & 0.616 & $5.5 \pm 0.3$ \\
Urine pH & & $6.3 \pm 0.6$ & $6.5 \pm 0.5$ & 0.203 & $6.4 \pm 0.6$
\end{tabular}

$p$ value: ANOVA.

(b)

\begin{tabular}{cccccc}
\hline \multirow{2}{*}{ Parameter } & LC-Plasma $(\mathrm{n}=20)$ & Placebo $(\mathrm{n}=20)$ & \multicolumn{2}{c}{ Whole $(\mathrm{n}=40)$} \\
\cline { 2 - 5 } & mean $\pm \mathrm{SD}$ & mean $\pm \mathrm{SD}$ & & mean $\pm \mathrm{SD}$ \\
\hline Urine protein & $0.00 \pm 0.00$ & $0.00 \pm 0.00$ & 1.000 & $0.00 \pm 0.00$ \\
Urine glucose & $0.00 \pm 0.00$ & $0.00 \pm 0.00$ & 1.000 & $0.00 \pm 0.00$ \\
Urobilinogen & $0.00 \pm 0.00$ & $0.00 \pm 0.00$ & 1.000 & $0.00 \pm 0.00$ \\
Urine bilirubin & $0.00 \pm 0.00$ & $0.00 \pm 0.00$ & 1.000 & $0.00 \pm 0.00$ \\
Urine ketone body & $0.00 \pm 0.00$ & $0.00 \pm 0.00$ & 1.000 & $0.00 \pm 0.00$ \\
Occult blood reaction & $0.05 \pm 0.22$ & $0.00 \pm 0.00$ & 0.317 & $0.03 \pm 0.16$ \\
\hline
\end{tabular}

$p$ value: Wilcoxon's rank sum test (asymptotic significance probability).

Table 2. Physical parameters at the start of intake, during the intake period and during follow up observations of all subjects.

\begin{tabular}{|c|c|c|c|c|c|c|c|c|c|c|c|c|c|c|}
\hline & & & & $0 \mathrm{~W}$ & & $2 \mathrm{~W}$ & & & $4 \mathrm{~W}$ & & & $6 \mathrm{~W}$ & & \\
\hline Parameter & $\begin{array}{l}\text { Reference } \\
\text { range }\end{array}$ & $\begin{array}{l}\text { Study } \\
\text { foods }\end{array}$ & $\mathrm{n}$ & mean $\pm \mathrm{SD}$ & $\begin{array}{c}p \\
\text { value } \\
1\end{array}$ & mean \pm SD & $\begin{array}{c}p \\
\text { value } \\
1 \\
\end{array}$ & $\begin{array}{c}p \\
\text { value } \\
2 \\
\end{array}$ & mean $\pm S D$ & $\begin{array}{c}p \\
\text { value } \\
1 \\
\end{array}$ & $\begin{array}{c}p \\
\text { value } \\
2 \\
\end{array}$ & mean $\pm S D$ & $\begin{array}{c}p \\
\text { value }^{f} \\
1\end{array}$ & $\begin{array}{c}p \text { value } \\
2\end{array}$ \\
\hline $\begin{array}{l}\text { Height } \\
(\mathrm{cm})\end{array}$ & & $\begin{array}{c}\text { LC-Plasma } \\
\text { Placebo }\end{array}$ & $\begin{array}{l}20 \\
20\end{array}$ & $\begin{array}{l}164.6 \pm 9.3 \\
165.1 \pm 8.7\end{array}$ & 0.849 & $\begin{array}{l}164.6 \pm 9.3 \\
165.1 \pm 8.7\end{array}$ & 0.869 & $\begin{array}{l}0.410 \\
0.841\end{array}$ & $\begin{array}{l}164.5 \pm 9.2 \\
165.2 \pm 8.7\end{array}$ & 0.808 & $\begin{array}{l}0.248 \\
0.529\end{array}$ & $\begin{array}{l}164.6 \pm 9.3 \\
165.1 \pm 8.7\end{array}$ & 0.860 & $\begin{array}{l}0.957 \\
0.487\end{array}$ \\
\hline $\begin{array}{l}\text { Body } \\
\text { weight } \\
(\mathrm{kg})\end{array}$ & & $\begin{array}{c}\text { LC-Plasma } \\
\text { Placebo }\end{array}$ & 20 & $\begin{array}{l}59.0 \pm 9.2 \\
59.9 \pm 7.7\end{array}$ & 0.726 & $\begin{array}{l}58.8 \pm 9.0 \\
59.7 \pm 7.5\end{array}$ & 0.735 & $\begin{array}{l}0.527 \\
0.054\end{array}$ & $\begin{array}{l}59.0 \pm 9.1 \\
59.8 \pm 7.7\end{array}$ & 0.774 & $\begin{array}{l}0.876 \\
0.516\end{array}$ & $\begin{array}{l}58.9 \pm 9.2 \\
59.6 \pm 7.5\end{array}$ & 0.793 & $\begin{array}{l}0.676 \\
0.079\end{array}$ \\
\hline BMI & & $\begin{array}{c}\text { LC-Plasma } \\
\text { Placebo }\end{array}$ & $\begin{array}{l}20 \\
20\end{array}$ & $\begin{array}{l}21.6 \pm 1.8 \\
21.9 \pm 1.8\end{array}$ & 0.622 & $\begin{array}{l}21.6 \pm 1.6 \\
21.9 \pm 1.8\end{array}$ & 0.599 & $\begin{array}{l}0.463 \\
0.109\end{array}$ & $\begin{array}{l}21.7 \pm 1.7 \\
21.9 \pm 1.9\end{array}$ & 0.756 & $\begin{array}{l}0.662 \\
0.393\end{array}$ & $\begin{array}{l}21.6 \pm 1.8 \\
21.8 \pm 1.9\end{array}$ & 0.728 & $\begin{array}{l}0.747 \\
0.085\end{array}$ \\
\hline $\begin{array}{l}\text { Systolic } \\
\text { blood } \\
\text { pressure } \\
(\mathrm{mmHg})\end{array}$ & $100-139$ & LC-Plasma & 20 & $\begin{array}{l}114.7 \pm 15.6 \\
117.0 \pm 11.8\end{array}$ & 0.603 & $\begin{array}{l}119.9 \pm 15.1 \\
119.7 \pm 11.1\end{array}$ & 0.953 & $\begin{array}{l}0.013 \\
0.263\end{array}$ & $\begin{array}{l}118.3 \pm 18.1 \\
114.9 \pm 12.8\end{array}$ & 0.503 & 0.083 & $\begin{array}{r}114.0 \pm 16.9 \\
114.8 \pm 9.4\end{array}$ & 0.845 & $\begin{array}{l}0.757 \\
0.311\end{array}$ \\
\hline
\end{tabular}




\section{Continued}

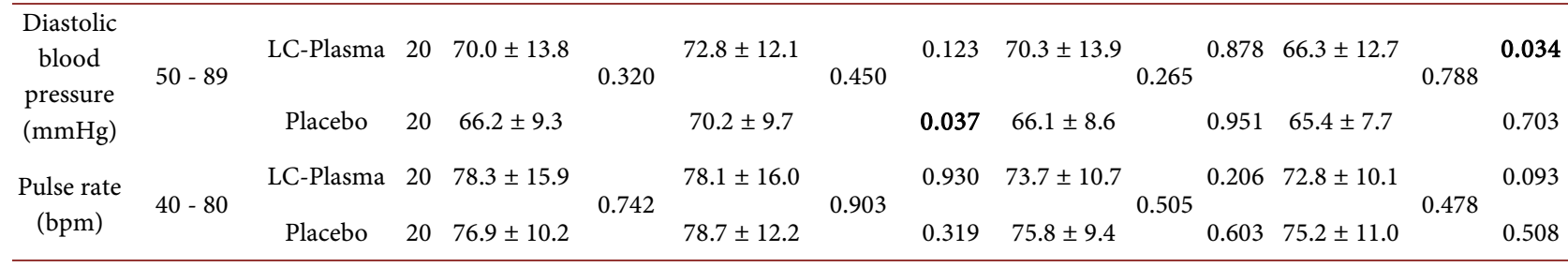

$p$ value 1: unpaired t-test (LC-Plasma group vs placebo group). $p$ value 2: paired t-test (vs $0 \mathrm{~W}$ ).

Table 3. Hematological parameters at the start of intake, during the intake period and during follow up observations of all subjects.

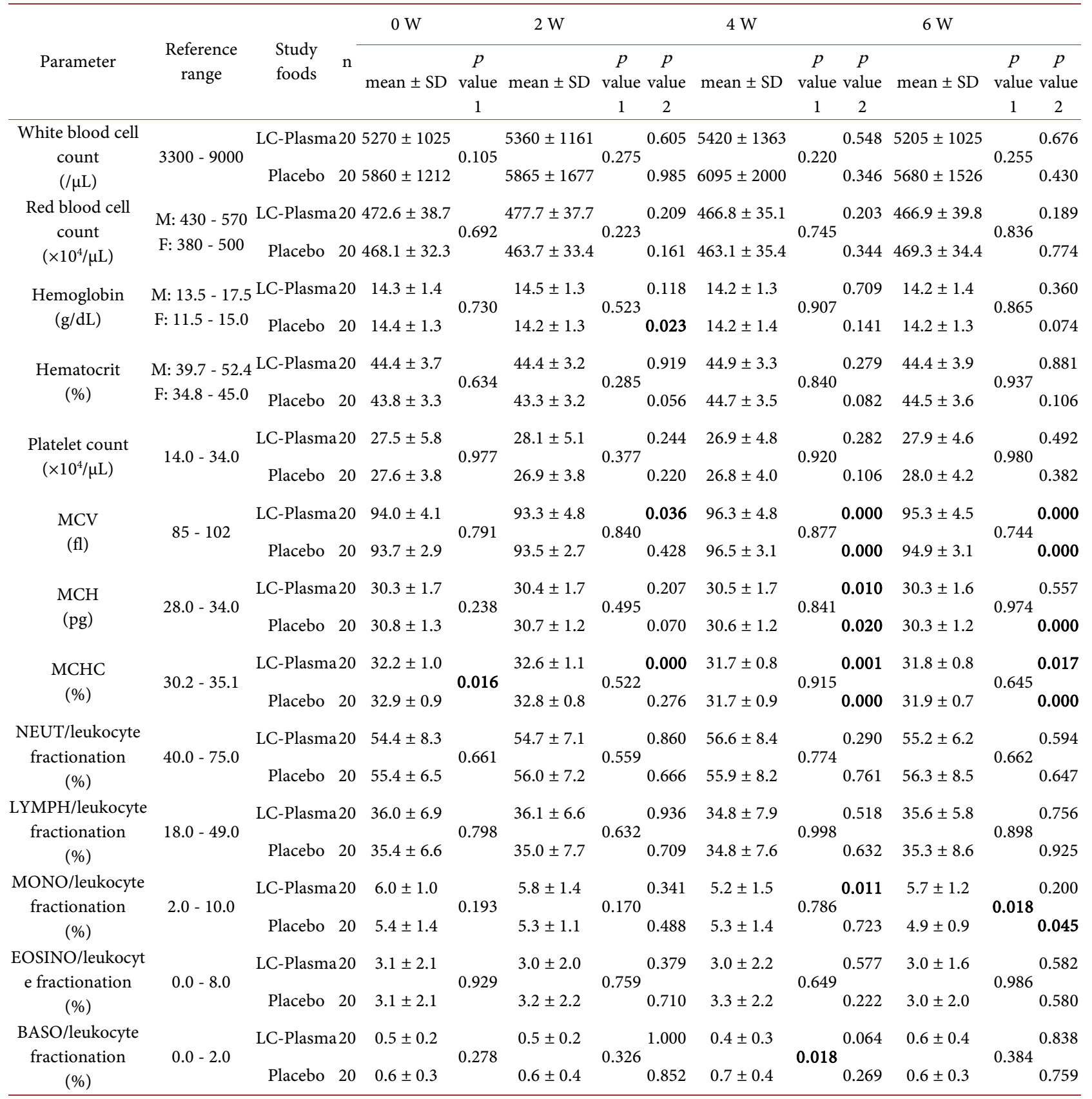

$p$ value 1: unpaired t-test (LC-Plasma group vs placebo group), $p$ value 2: paired t-test (vs $0 \mathrm{~W}$ ). 
Table 4. Hematological parameters at the start of intake, during the intake period and during the follow up observations for male and female subjects.

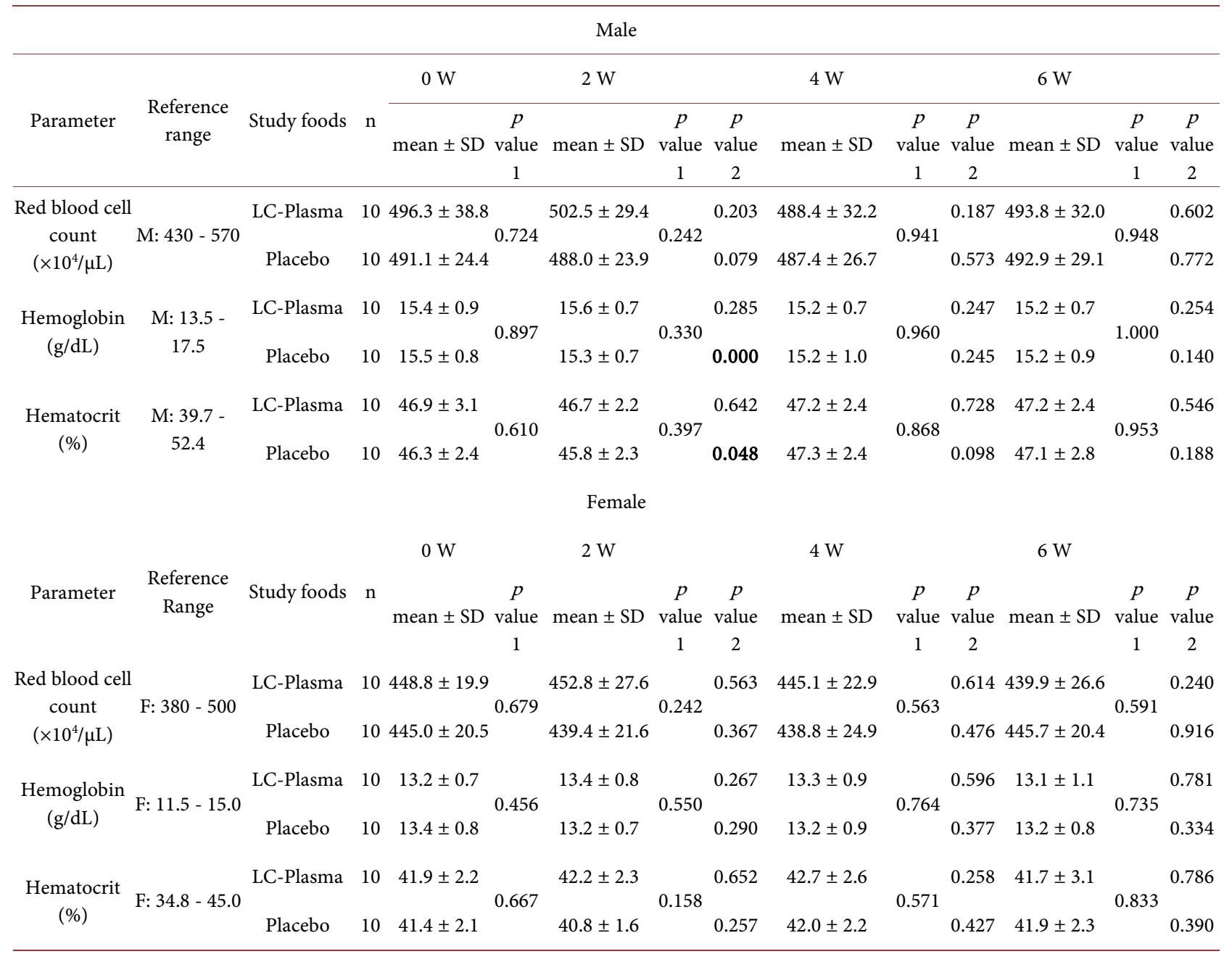

$p$ value 1: unpaired t-test (LC-Plasma group vs placebo group). $p$ value 2: paired t-test (vs $0 \mathrm{~W}$ ).

Table 5. Biochemical parameters at the start of intake, during the intake period and during follow up observations for all subjects.

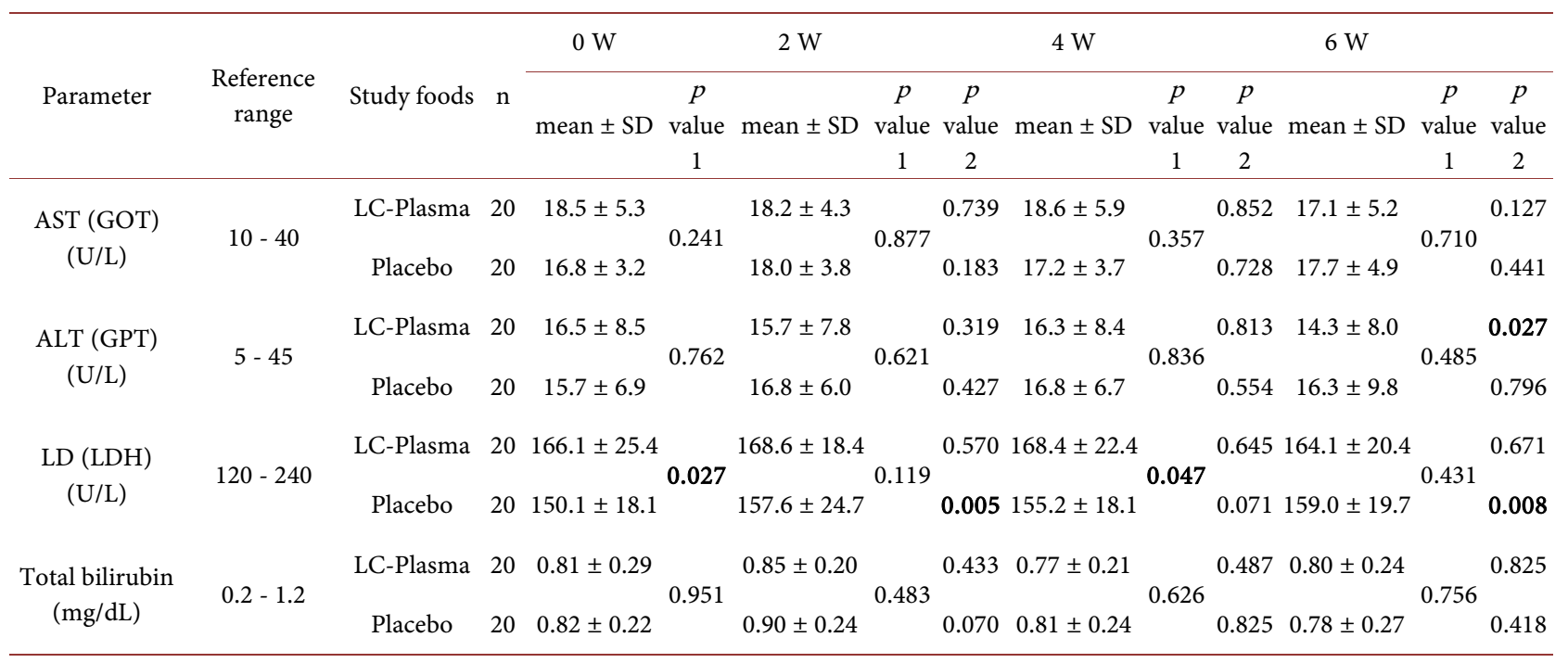




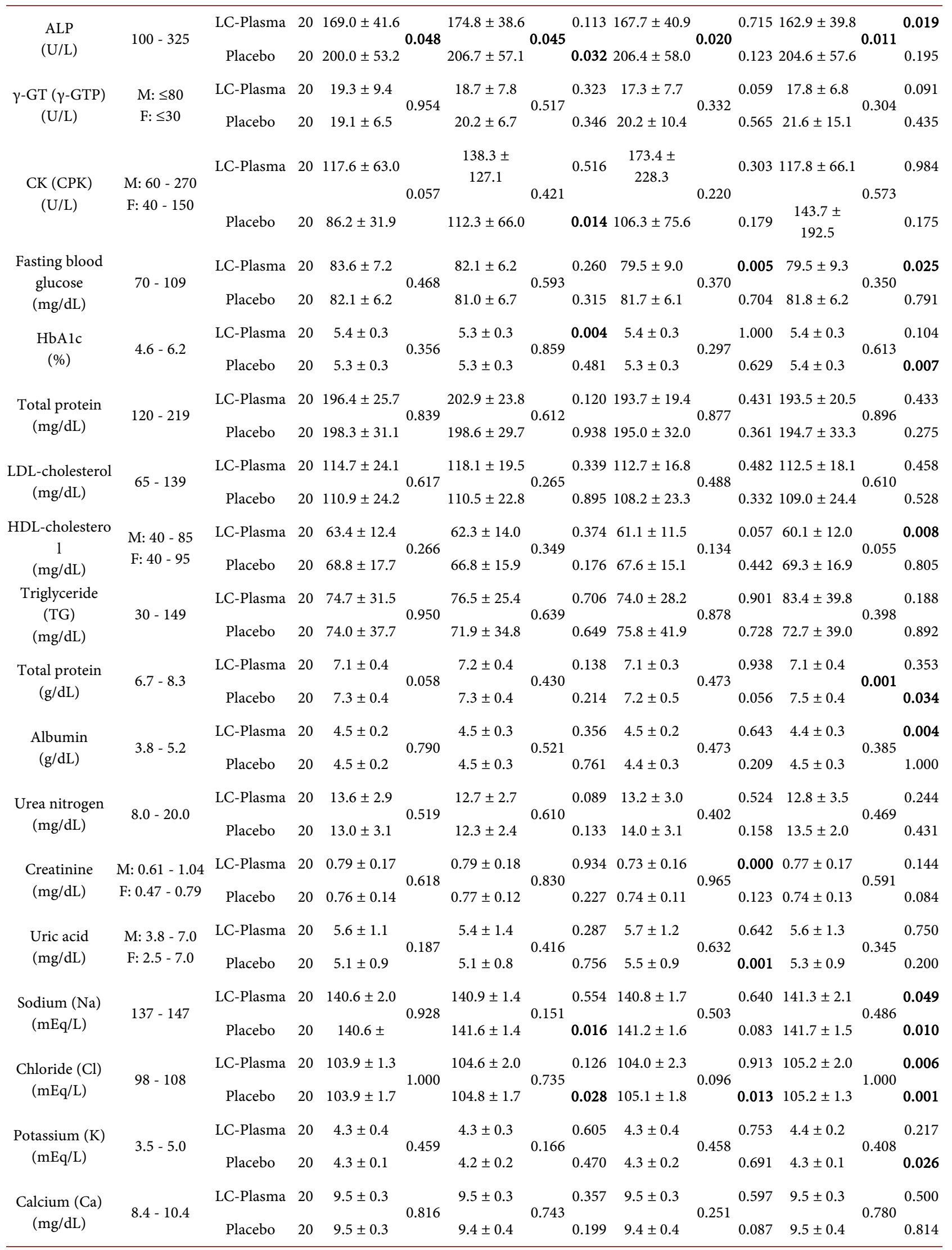




\section{Continued}

\begin{tabular}{|c|c|c|c|c|c|c|c|c|c|c|c|c|c|c|}
\hline Inorganic & & LC-Plasma & 20 & $3.6 \pm 0.5$ & & $3.5 \pm 0.6$ & & 0.607 & $3.6 \pm 0.5$ & & 0.834 & $3.4 \pm 0.5$ & & 0.084 \\
\hline $\begin{array}{l}\text { phosphorus } \\
(\mathrm{mg} / \mathrm{dL})\end{array}$ & $2.5-4.5$ & Placebo & 20 & $3.8 \pm 0.5$ & 0.257 & $3.6 \pm 0.4$ & 0.527 & 0.177 & $3.7 \pm 0.4$ & 0.384 & 0.496 & $3.7 \pm 0.6$ & 0.099 & 0.771 \\
\hline $\begin{array}{c}\text { Magnesium } \\
(\mathrm{mg} / \mathrm{dL})\end{array}$ & $1.9-2.5$ & $\begin{array}{c}\text { LC-Plasma } \\
\text { Placebo }\end{array}$ & $\begin{array}{l}20 \\
20\end{array}$ & $\begin{array}{l}2.1 \pm 0.1 \\
2.1 \pm 0.1\end{array}$ & 0.575 & $\begin{array}{l}2.2 \pm 0.1 \\
2.2 \pm 0.1\end{array}$ & 0.350 & $\begin{array}{l}0.004 \\
0.000\end{array}$ & $\begin{array}{l}2.1 \pm 0.1 \\
2.2 \pm 0.1\end{array}$ & 0.225 & $\begin{array}{l}0.163 \\
0.001\end{array}$ & $\begin{array}{l}2.1 \pm 0.1 \\
2.1 \pm 0.1\end{array}$ & 0.770 & $\begin{array}{l}1.000 \\
0.772\end{array}$ \\
\hline
\end{tabular}

$\mathrm{p}$ value 1: unpaired t-test (LC-Plasma group vs placebo group). $\mathrm{p}$ value 2: paired t-test (vs $0 \mathrm{~W}$ ).

Table 6. Biochemical parameters at the start of intake, during the intake period and during follow up observations for male and female subjects.

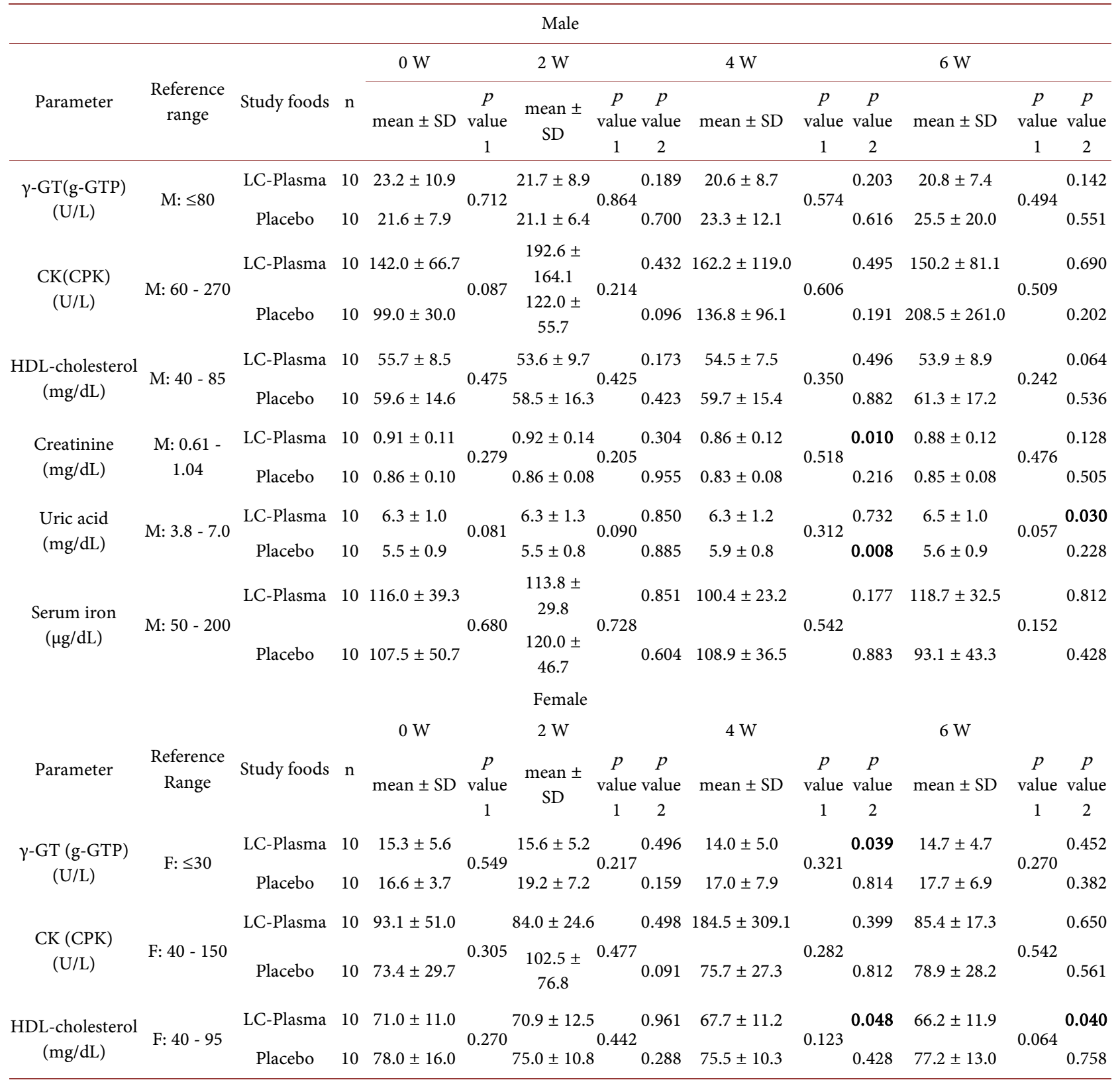




\section{Continued}

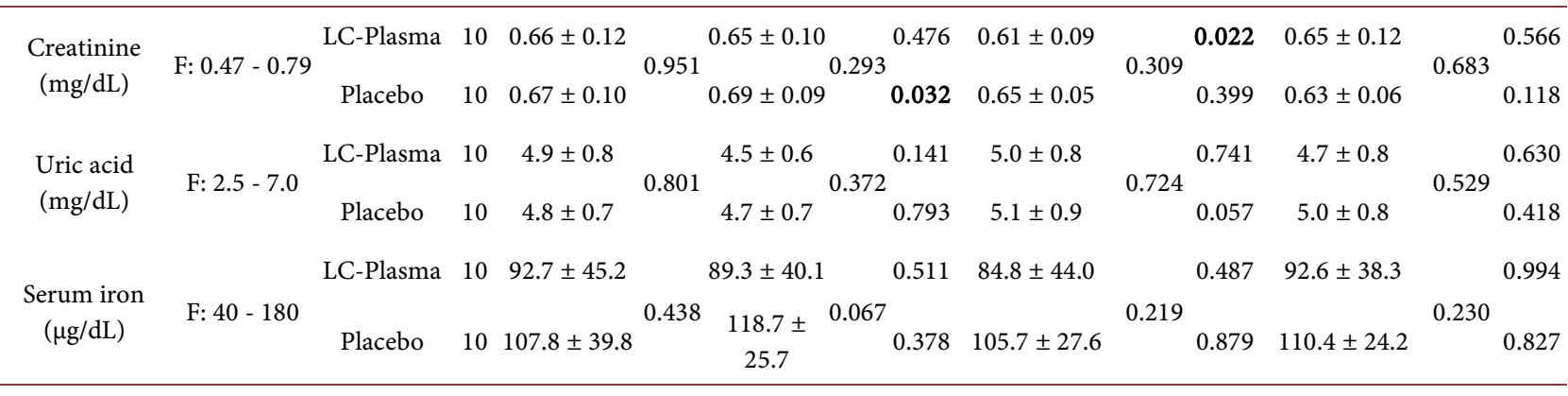

$p$ value 1: unpaired t-test (LC-Plasma group vs placebo group). $p$ value 2: paired t-test (vs $0 \mathrm{~W}$ ).

Table 7. Urinalysis parameters at the start of intake, during the intake period and during follow up observations for all subjects.

\begin{tabular}{|c|c|c|c|c|c|c|c|c|c|c|c|c|c|}
\hline \multirow{3}{*}{ Parameter } & \multirow{3}{*}{ Study Foods } & \multirow{3}{*}{$\mathrm{n}$} & \multicolumn{2}{|l|}{$0 \mathrm{~W}$} & \multicolumn{3}{|l|}{$2 \mathrm{~W}$} & \multicolumn{3}{|l|}{$4 \mathrm{~W}$} & \multicolumn{3}{|l|}{$6 \mathrm{~W}$} \\
\hline & & & mean $\pm \mathrm{SD}$ & $\begin{array}{c}p \\
\text { value }\end{array}$ & mean $\pm S D$ & $p$ value & $p$ value & mean $\pm S D$ & $\begin{array}{c}p \\
\text { value }\end{array}$ & $\begin{array}{c}p \\
\text { value }\end{array}$ & mean $\pm \mathrm{SD}$ & $\begin{array}{c}p \\
\text { value }\end{array}$ & $\begin{array}{c}p \\
\text { value }\end{array}$ \\
\hline & & & & 1 & & & & & 1 & 2 & & 1 & 2 \\
\hline \multirow{2}{*}{ Urine protein } & LC-Plasma & 20 & $0.20 \pm 0.70$ & \multirow{2}{*}{0.534} & $0.10 \pm$ & \multirow{2}{*}{0.317} & 0.157 & $0.00 \pm 0.00$ & \multirow{2}{*}{0.317} & 0.180 & $0.00 \pm 0.00$ & \multirow{2}{*}{1.000} & 0.180 \\
\hline & Placebo & 20 & $0.05 \pm 0.22$ & & $0.00 \pm 0.00$ & & 0.317 & $0.05 \pm 0.22$ & & 1.000 & $0.00 \pm 0.00$ & & 0.317 \\
\hline \multirow[b]{2}{*}{ Urine glucose } & LC-Plasma & 20 & $0.00 \pm 0.00$ & \multirow[b]{2}{*}{1.000} & $0.00 \pm 0.00$ & \multirow[b]{2}{*}{1.000} & 1.000 & $0.00 \pm 0.00$ & \multirow[b]{2}{*}{1.000} & 1.000 & $0.00 \pm 0.00$ & \multirow[b]{2}{*}{1.000} & 1.000 \\
\hline & Placebo & 20 & $0.00 \pm 0.00$ & & $0.00 \pm 0.00$ & & 1.000 & $0.00 \pm 0.00$ & & 1.000 & $0.00 \pm 0.00$ & & 1.000 \\
\hline \multirow{2}{*}{ Urobilinogen } & LC-Plasma & 20 & $0.00 \pm 0.00$ & \multirow{2}{*}{1.000} & $0.00 \pm 0.00$ & \multirow{2}{*}{1.000} & 1.000 & $0.00 \pm 0.00$ & \multirow{2}{*}{1.000} & 1.000 & $0.00 \pm 0.00$ & \multirow{2}{*}{1.000} & 1.000 \\
\hline & Placebo & 20 & $0.00 \pm 0.00$ & & $0.00 \pm 0.00$ & & 1.000 & $0.00 \pm 0.00$ & & 1.000 & $0.00 \pm 0.00$ & & 1.000 \\
\hline \multirow{2}{*}{ Urine bilirubin } & LC-Plasma & 20 & $0.00 \pm 0.00$ & \multirow{2}{*}{1.000} & $0.00 \pm 0.00$ & \multirow{2}{*}{1.000} & 1.000 & $0.00 \pm 0.00$ & \multirow{2}{*}{1.000} & 1.000 & $0.00 \pm 0.00$ & \multirow[b]{2}{*}{1.000} & 1.000 \\
\hline & Placebo & 20 & $0.00 \pm 0.00$ & & $0.00 \pm 0.00$ & & 1.000 & $0.00 \pm 0.00$ & & 1.000 & $0.00 \pm 0.00$ & & 1.000 \\
\hline \multirow{2}{*}{$\begin{array}{l}\text { Urine ketone } \\
\text { body }\end{array}$} & LC-Plasma & 20 & $0.00 \pm 0.00$ & \multirow{2}{*}{1.000} & $0.00 \pm 0.00$ & \multirow{2}{*}{1.000} & 1.000 & $0.20 \pm 0.89$ & \multirow{2}{*}{0.317} & 0.317 & $0.00 \pm 0.00$ & \multirow{2}{*}{1.000} & 1.000 \\
\hline & Placebo & 20 & $0.00 \pm 0.00$ & & $0.00 \pm 0.00$ & & 1.000 & $0.00 \pm 0.00$ & & 1.000 & $0.00 \pm 0.00$ & & 1.000 \\
\hline \multirow{2}{*}{$\begin{array}{l}\text { Occult blood } \\
\text { reaction }\end{array}$} & LC-Plasma & 20 & $0.20 \pm 0.89$ & \multirow{2}{*}{0.317} & $0.15 \pm 0.67$ & \multirow{2}{*}{0.971} & 0.655 & $0.20 \pm 0.89$ & & 1.000 & $0.00 \pm 0.00$ & 015 & 0.317 \\
\hline & Placebo & 20 & $0.00 \pm 0.00$ & & $0.10 \pm 0.45$ & & 0.317 & $0.05 \pm 0.22$ & & 0.317 & $0.10 \pm 0.31$ & & 0.157 \\
\hline
\end{tabular}

$p$ value 1: Wilcoxon's rank sum test (LC-Plasma group vs placebo group) asymptotic significance probability. $p$ value 2: Wilcoxon's signed rank test (vs $0 \mathrm{~W}$ ) asymptotic significance probability.

Table 8. Urine $\mathrm{pH}$ and urine specific gravity at the start of intake, during the intake period and during follow up observations for all subjects.

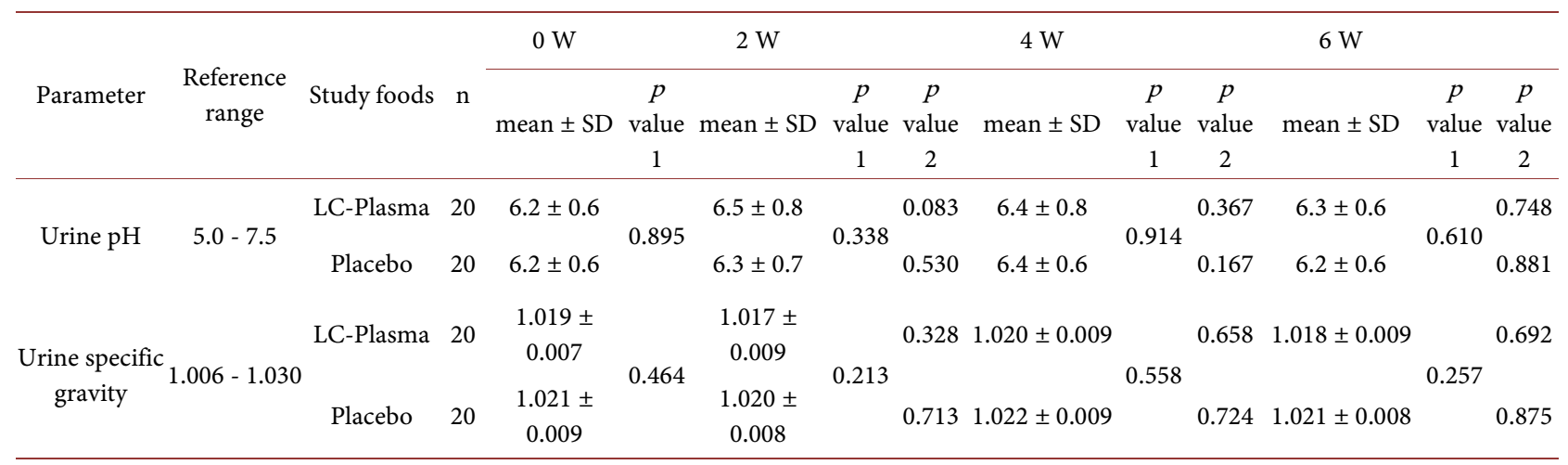

$p$ value 1: unpaired t-test (LC-Plasma group vs placebo group). $p$ value 2: paired t-test (vs $0 \mathrm{~W}$ ). 


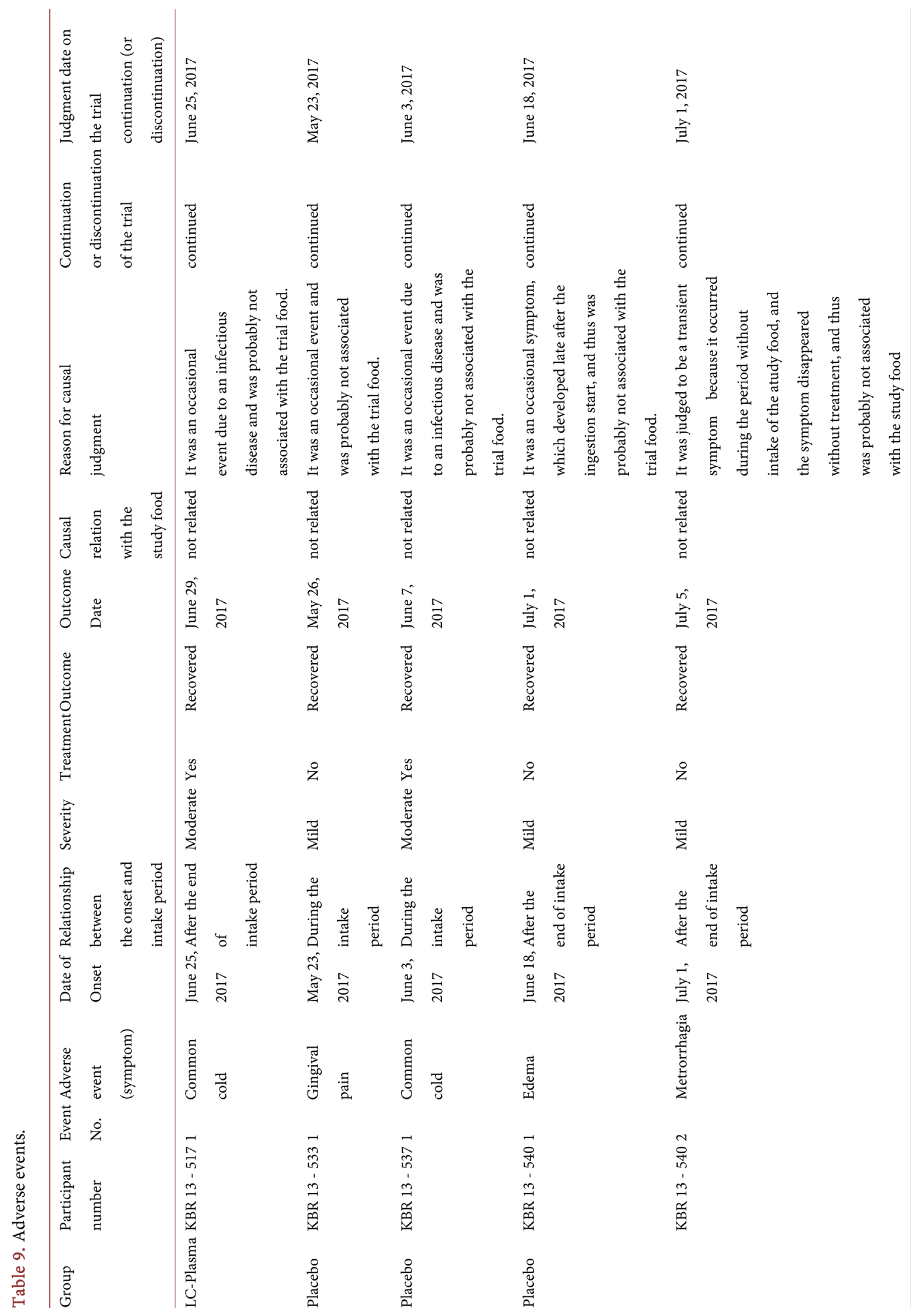


differed between males and females (Table 4 and Table 6). Data for the qualitative evaluation parameters were scored and basic statistics were calculated. Statistical analysis was performed by Wilcoxon's signed rank test for comparison of the data obtained at the start of ingestion (in week 0), and those obtained at the examination in weeks 2, 4 (intake period) and 6 (at the end of the follow up). In addition, statistical analysis was conducted by Wilcoxon's rank sum test for comparison of the data at each examination between the LC-Plasma group and placebo group (Table 7). All statistical tests were conducted with a significance level of 0.05 .

\section{Results}

Subject characteristics. The Consolidated Standards of Reporting Trials flow diagram for this trial is shown in Figure 1. A total of 103 healthy volunteers were initially recruited. Fifty eight subjects were excluded based on the exclusion criteria, three subjects refused to participate and two subjects were not selected.

Table 1(a) and Table 1(b) show the baseline characteristics of each group. A significant difference between the LC-Plasma group and the placebo group was observed in the levels of LD (LDH), however, this fluctuation in a single parameter is negligible since the primary purpose of the trial is to evaluate the overall safety of excessive intake of LC-Plasma capsules. The trial was conducted as illustrated in Table 1(a) and Table 1(b). None of the subjects met the predefined discontinuance criteria during the examination period, and thus data obtained from all 40 subjects were included in the analysis.

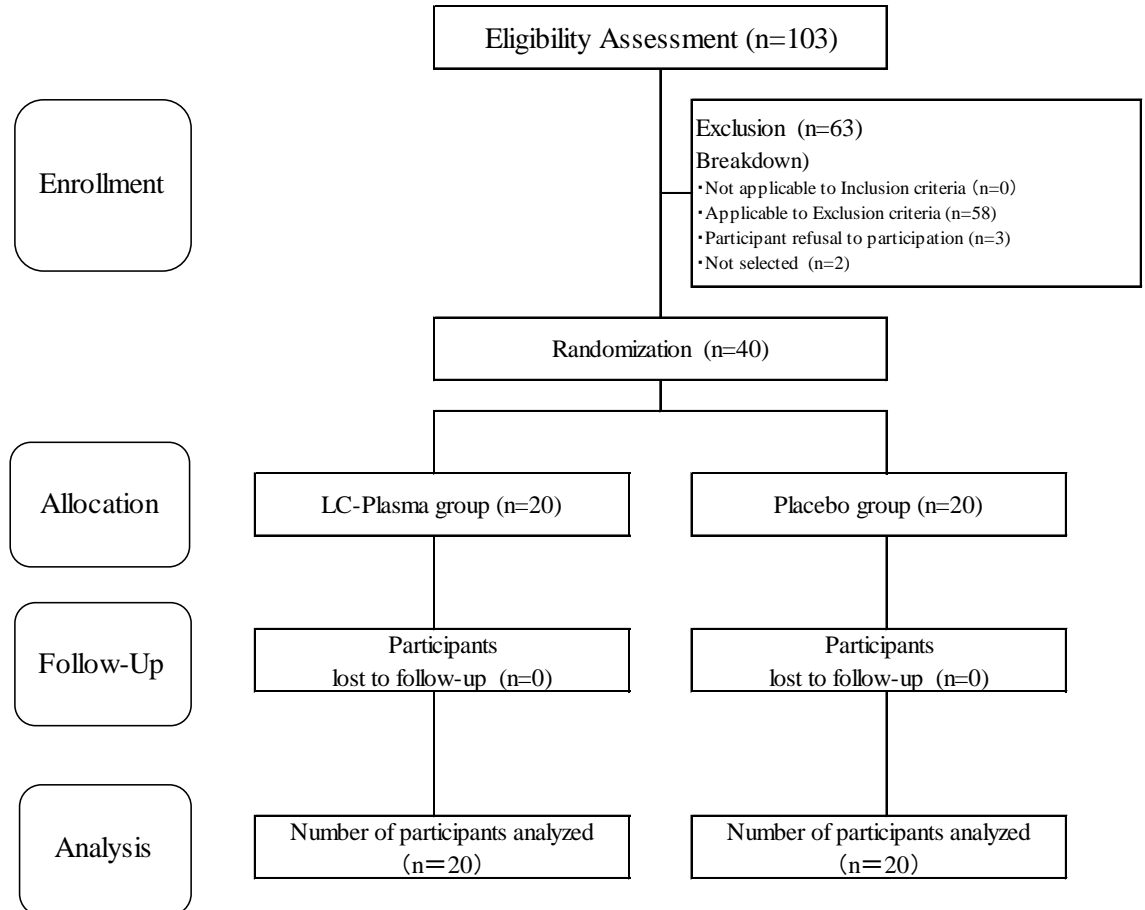

Figure 1. Consolidated standards of reporting trials diagram: Enrollment, random assignment, and follow-up of subjects. 
Physical examinations: The results of the physical examinations are shown in Table 2. Comparing the values to week 0 , significant changes were observed in the following parameters: in the LC-Plasma group, higher systolic blood pressure in week 2, lower diastolic blood pressure in week 6; in the placebo group, higher diastolic blood pressure in week 2 . No significant changes were observed in the other parameters. Although significant changes were observed among several parameters in the physical examinations during the trial, the changes were within the range of physiological fluctuation and were deemed clinically irrelevant. Based on the physical measurements, this trial was judged to be of no safety concern by the principal investigator.

Hematological examinations. The results of the hematological examinations of all subjects are shown in Table 3. Comparing the values in week 0 , significant changes were observed in the following parameters: in the LC-Plasma group, lower MCV in week 2, higher MCV in weeks 4 and 6 , higher MCH in week 4, higher MCHC in week 2, lower MCHC in weeks 4 and 6, lower MONO/leukocyte fractionation in week 4; in the placebo group, lower hemoglobin in week 2, higher MCV in weeks 4 and 6, lower MCH in weeks 4 and 6, lower MCHC in weeks 4 and 6, lower $\mathrm{MONO}$ /leukocyte fractionation in week 6. Compared to the placebo group, significant changes were observed in the LC-Plasma group in the following parameters: lower MCHC at the start of ingestion; higher $\mathrm{MONO} /$ leukocyte fractionation in week 6; lower BASO/leukocyte fractionation in week 4 . The results of the hematological examinations in male and female subjects are shown in Table 4. In the male group, compared to the values in week 0 , significant changes were observed in the following parameters: in the placebo group, lower hemoglobin in week 2, lower hematocrit in week 2. No significant changes were observed in the female group. Although significant changes were observed among several parameters in the hematological examinations during the trial, these changes were within the range of physiological fluctuation and deemed clinically irrelevant. Based on the hematological measurements, this trial was judged to be of no safety concern by the principal investigator.

Biochemical examinations: The results of the biochemical examinations of all subjects are shown in Table 5. Compared to the values in week 0 , significant changes were observed in the following parameters: in the LC-Plasma group, lower ALT (GPT) in week 6, lower ALP in week 6, lower fasting blood glucose in weeks 4 and 6, lower HbAlc in week 2, lower HDL-cholesterol in week 6, lower albumin in week 6 , lower creatinine in week 4 , higher sodium $(\mathrm{Na})$ in week 6 , higher chloride $(\mathrm{Cl})$ in week 6 , higher magnesium in week 2; in the placebo group, higher LD (LDH) in weeks 2 and 6, higher ALP in week 2, higher CK (CPK) in week 2, higher HbAlc in week 6, higher total protein in week 6, higher uric acid in week 4 , higher sodium $(\mathrm{Na})$ in week 2 and in week 6 , higher chloride $(\mathrm{Cl})$ in week 2 , in week 4 and in week 6 , higher potassium (K) in week 6 , higher magnesium in weeks 2 and 4 . Compared to the placebo group, significant changes were observed in the LC-Plasma group in the following parameters: 
higher $\mathrm{LD}(\mathrm{LDH})$ at the start of ingestion and week 4; lower ALP at the start of ingestion and weeks 2, 4 and 6; lower total protein in week 6. The results of the biochemical examinations of male or female subjects are shown in Table 6. In the male group, compared to the values in week 0 , significant changes were observed in the following parameters: in the LC-Plasma group, lower creatinine in week 4, higher uric acid in week 6; in the placebo group, higher uric acid in week 4. In the female group, compared to the values in week 0 , significant changes were observed in the following parameters: in the LC-Plasma group, lower $\gamma$-GT (g-GTP) in week 4, lower HDL-cholesterol in weeks 4 and 6, lower creatinine in week 4; in the placebo group, higher creatinine in week 2 . No significant changes were observed in the other parameters. Although significant changes were observed among several parameters in the biochemical examinations during the trial, these changes were within the range of physiological fluctuation and deemed clinically irrelevant. Based on the biochemical measurements, this was judged to be of no safety concern by the principal investigator.

Urinalysis examinations. The results of the urinalysis (quantitative test and scoring qualitative test) are shown in Table 7 and Table 8. No significant changes were observed in any of the parameters.

Adverse events. Five adverse events (one event in one subject in the LC-Plasma group and four events in three subjects in the placebo group) were observed during the trial period (Table 9). The principal investigator judged that all of the adverse events were "not related" to the trial capsules because the events were accidental or occurred outside of the intake period.

\section{Discussion}

Lactococcus lactis has been traditionally used in foods such as cheese and has been listed in "Qualified Presumption of Safety (QPS)-recommended biological agents intentionally added to food or feed" issued by the European Food Safety Authority [13]. Pharmacokinetics of LC-Plasma is thought that orally administrated LC-Plasma are taken up by immune cells in Peyer's patches of the intestinal tract and then induce expression of IFN- $\alpha$ [6]. LC-Plasma which are not taken up by immune cells are thought to be excreted in the feces. Previous pre-clinical studies in rats showed that single oral administration of $2000 \mathrm{mg} / \mathrm{kg}$ of LC-Plasma to 6-week-old Sprague-Dawley rats had no toxicity in general conditions, changes in body weight or necropsy, in addition that no toxicity was associated with repeated administration of $3.0 \times 10^{10}$ or $3.0 \times 10^{11} \mathrm{cfu} / \mathrm{kg}$ of LC-Plasma once a day for 28 days to 6-week-old Sprague-Dawley rats: measurements of body weight, urinalysis, hematological and blood biochemical examinations, histopathological examination of major organs and observations on the general condition of the rats were all judged to be within the range of physiological fluctuation. In micronucleus test, LC-Plasma was considered not to induce micronuclei in mouse peripheral blood with oral administrations of 500 $\mathrm{mg} / \mathrm{kg}, 1000 \mathrm{mg} / \mathrm{kg}$ or $2000 \mathrm{mg} / \mathrm{kg}$ of LC-Plasma twice in consecutive 24-hour 
intervals to 7-week-old ICR mice. Previous clinical trials also showed that the intake of a beverage containing $150 \mathrm{mg} /$ day LC-Plasma (3 times the anti-viral infection effect dose) and $15 \mathrm{~g} /$ day indigestible dextrin for four weeks and intake of a beverage containing $50 \mathrm{mg} /$ day LC-Plasma (anti-viral effective dose) and 5 $\mathrm{g} /$ day indigestible dextrin for twelve weeks were safe [14]. Moreover, products containing LC-Plasma have been marketed as soft drinks and yogurt from Kirin Beverage Co., Ltd. and Koiwai Milk Products Co., Ltd, and no health damage associated with them has been reported.

In this trial, the safety of ingesting LC-Plasma $(250 \mathrm{mg} /$ day $)$ for four weeks was assessed in forty subjects. The examinations included physical, hematological, biochemical and urinary examinations and a medical interview. In the urinary examinations, there were no significant changes within the LC-Plasma group and the placebo group when compared to the reference values (week 0) and no significant changes between the LC-Plasma group and the placebo group. Significant differences and measurements outside the reference range were found in various parameters of the physical, hematological and biochemical examinations but none were considered to be a safety concern by the principal investigator. Significant differences in various parameters were also found between the LC-Plasma group and the placebo group, however, all of these differences were within the range of the reference values and within the range of physiological fluctuation. Thus, these differences were not considered to be a clinical safety concern attributable to the intake of the LC-Plasma capsules. The investigator determined that the adverse events noted in medical interviews by physicians and diary records by subjects were not related to the trial capsules because all of them were accidental or occurred outside of the intake period. Therefore, it was concluded that there are no clinical safety concerns associated with the excessive intake (5 times the anti-viral effective dose) of LC-Plasma.

\section{Conclusion}

There are no safety concerns associated with the consumption of heat-killed LC-Plasma ( $250 \mathrm{mg} /$ day) for four weeks in the results obtained from physical, hematological, biochemical and urinary examinations and medical interviews in a randomized double-blind placebo-controlled parallel-group comparison method using 40 healthy adult males and females.

\section{References}

[1] Takeda, K., Suzuki, T., Shimada, S.I., Shida, K., Nanno, M. and Okumura, K. (2006) Interleukin-12 Is Involved in the Enhancement of Human Natural Killer Cell Activity by Lactobacillus casei Shirota. Clinical \& Experimental Immunology, 146, 109-115. https://doi.org/10.1111/j.1365-2249.2006.03165.x

[2] Kanauchi, O., Andoh, A., Abu Bakar, S. and Yamamoto, N. (2018) Probiotics and Paraprobiotics in Viral Infection: Clinical Application and Effects on the Innate and Acquired Immune Systems. Current Pharmaceutical Design, 24.

[3] Jounai, K., Ikado, K., Sugimura, T., Ano, Y., Braun, J. and Fujiwara, D. (2012) 
Spherical Lactic Acid Bacteria Activate Plasmacytoid Dendritic Cells Immunomodulatory Function via TLR9-Dependent Crosstalk with Myeloid Dendritic Cells. PLoS ONE, 7, e32588. https://doi.org/10.1371/journal.pone.0032588

[4] Siegal, F.P., Kadowaki, N., Shodell, M., Fitzgerald-Bocarsly, P.A., Shah, K., Ho, S., Antonenko, S. and Liu, Y. (1999) The Nature of the Principal Type 1 Interferon-Producing Cells in Human Blood. Science, 284, 1835-1837.

https://doi.org/10.1126/science.284.5421.1835

[5] Cella, M., Jarrossay, D., Facchetti, F., Alebardi, O., Nakajima, H., Lanzavecchia, A. and Colonna, M. (1999) Plasmacytoid Monocytes Migrate to Inflamed Lymph Nodes and Produce Large Amounts of Type I Interferon. Nature Medicine, 5, 919-923. https://doi.org/10.1038/11360

[6] Jounai, K., Sugimura, T., Morita, Y., Ohshio, K. and Fujiwara, D. (2015) Oral Administration of Lactococcus lactis Subsp. lactis JCM5805 Enhances Lung Immune Response Resulting in Protection from Murine Parainfluenza Virus Infection. PLoS ONE, 10, e0119055. https://doi.org/10.1371/journal.pone.0119055

[7] Jounai, K., Sugimura, T., Morita, Y., Ohshio, K. and Fujiwara, D. (2018) Administration of Lactococcus lactis Strain Plasma Induces Maturation of Plasmacytoid Dendritic Cells and Protection from Rotavirus Infection in Suckling Mice. International Immunopharmacology, 56, 205-211. https://doi.org/10.1016/j.intimp.2018.01.034

[8] Sugimura, T., Takahashi, H., Jounai, K., Ohshio, K., Kanayama, M., Tazumi, K., Tanihata, Y., Miura, Y., Fujiwara, D. and Yamamoto, N. (2015) Effects of Oral Intake of Plasmacytoid Dendritic Cells-Stimulative Lactic Acid Bacterial Strain on Pathogenesis of Influenza-Like Illness and Immunological Response to Influenza Virus. British Journal of Nutrition, 114, 727-733. https://doi.org/10.1017/S0007114515002408

[9] Sugimura, T., Jounai, K., Ohshio, K., Tanaka, T., Suwa, M. and Fujiwara, D. (2013) Immunomodulatory Effect of Lactococcus lactis JCM5805 on Human Plasmacytoid Dendritic Cells. Clinical Immunology, 149, 509-518.

https://doi.org/10.1016/j.clim.2013.10.007

[10] Shibata, T., Kanayama, M., Haida, M., Fujimoto, S., Oroguchi, T., Sata, K., Mita, N., Kutsuzawa, T., Ikeuchi, M., Kondo, M., Naito, K., Tsuda, M., Nishizaki, Y. and Ishii, N. (2016) Lactococcus lactis JCM5805 Activates Anti-Viral Immunity and Reduces Symptoms of Common Cold and Influenza in Healthy Adults in a Randomized Controlled Trial. Journal of Functional Foods, 24, 492-500.

https://doi.org/10.1016/j.jff.2016.03.035

[11] Fujii, T., Jounai, K., Horie, A., Takahashi, H., Suzuki, H., Ohshio, K., Fujiwara, D. and Yamamoto, N. (2017) Effects of Heat-Killed Lactococcus lactis subsp. lactis JCM 5805 on Mucosal and Systemic Immune Parameters, and Antiviral Reactions to Influenza Virus in Healthy Adults; A Randomized Controlled Double-Blind Study. Journal of Functional Foods, 35, 513-521. https://doi.org/10.1016/j.jff.2017.06.011

[12] Japan Health Food \& Nutrition Food Association (2008) The Current Status of Safety Assessment of Food for Specified Health Use.

[13] EFSA Panel on Biological Hazards (BIOHAZ) (2015) Statement on the Update of the List of QPS-Recommended Biological Agents Intentionally Added to Food or Feed as Notified to EFSA. 2: Suitability of Taxonomic Units Notified to EFSA until March 2015. EFSA Journal, 13, 4138. https://doi.org/10.2903/j.efsa.2015.4138

[14] Tanaka, K., Suzuki, H., Kanayama, M., Fujii, T., Fujiwara, D., Nozawa, H. and Sugimura, H. (2015) Jpn Pharmacol Ther, 43, 1711-1727. 